\title{
सुरबहार की ऐतिहासिक पृष्ठभूमि
}

ARSHI

Research Scholar, Deptt. of Music, Panjab University, Chandigarh

\begin{abstract}
One of the important instruments to appear in late 19th century Hindustan was the surbahar, Hindi-Urdu "springtime of notes", an enlarged version of the kachua, flat gourd, sitar. The deep long-lasting sound of this instrument and its capability for extensive pulling of the main string made it suitable for playing music closely imitative of the been.The Surbahar is meant only for Alap and the alap should be executed like Veena's thirteen (alap) divisions. Playing of gats like Maseet Khani and Razakhani is strictly prohibited in Surbahar. The expositions of Thumri-ang ragas is also not meant for Surbahar and hence the use of Khatkas and Murkees is also prohibited but in present time, some instrumentalist uses all the alankars like Khatka Murki etc. of Khyal in Surbahar. So consequently Taans and Paltas can use in Surbahar too and the style became different from the orthodox Surbahar technique which followed the Veena ang strictly.
\end{abstract}

ABSTRACT

पृष्ठभूमि

'सुरबहार' नामक वाद्य भारतीय शास्त्रीय संगीत में 19वीं सदी के प्रारम्भिक वर्षों में प्रविष्ट हुआ। कानपुर के पास लखनऊ में सन् 1825 के लगभग सुरबहार के आविष्कार का पता चलता है। 'सुरबहार' अर्थात् वह वाद्य जिसमें स्वरों की बहार हो, वही सुरबहार है। सुरबहार के आविष्कर्ता के विषय में आचार्यों के भिन्न-भिन्न मत हैं।

एस. एम. टैगोर के अनुसार ${ }^{3}$

19वीं शताब्दी के आरम्भ में सन् 1830 के लगभग भारत में सुरबहार का प्रादुर्भाव हुआ। हाँलाकि इसकी मूलभूत बनावट सितार की भाँति ही थी, किन्तु इसका आकार सितार से काफी बड़ा था और सितार के अतिरिक्त इसमें कई खूबियां हैं। सेनिया घराने के संगीतज्ञ बीन या रबाब किसी बाहरी व्यक्ति को नहीं सिखाते थे। अतः प्रतिभाशाली विद्यार्थी जो इनके रिश्तेदार नहीं थे वे इस शेली को नहीं सीख सकते थे। कुछ विद्वानों के अनुसार लखनऊ के बीनकार उमराव ख़ां जो अपनी बेटी की तरफ से तानसेन घराने से संबंधित थे, ने एक बड़ा सितार बनाया था और उसका नाम सुरबहार रखा था और अपने खास विद्यार्थियों को ध्रुपद अंग में आलाप और जोड़ालाप सिखाते थे, उन्हीं में एक गुलाम मोहम्मद थे।

सुरबहार का आविष्कार बाँदा के मशहूर सितार और सुरबहार वादक उमराव ख़ां के शिष्य गुलाम मोहम्मद ने किया था। गुलाम मोहम्मद ने वाद्य-यंत्र निर्माताओं की सहायता से निराले ढंग का नया सितार बनाया था। सन् 1876 में रहीम बेग ने अपनी पुस्तक 'नग़मा-ए-सितार' में इसका चित्रण किया है। इस चित्र में, इस वाद्य-यंत्र में पांच तार, एक चौड़ी गर्दन, दस तरह के तार और एक अतिरिक्त तुम्बा दर्शाया गया है। परन्तु निश्चित रूप से नहीं कहा जा सकता है कि यह सुरबहार है या सितार की ही कोई किरम है।

1 Allyn Miner, Sitar and Sarod in the 18th and 19th Centuries, Page - 54

2 Allyn Miner, Sitar and Sarod in the 18th and 19th Centuries, Page - 54

3 Surinder Mohan Tagore, Yantra Kosh, Page - 17, 20, 21 \& 32 
सम्भवतःउमराव ख़ां ने ही इस यंत्र का आविष्कार किया था लेकिन चूँकि उन्होंने इसका वादन स्वयं नहीं किया और गुलाम मोहम्मद को बजाना सिखा दिया, इसलिए इसको प्रकाश में लाने और लोकप्रिय बनाने का श्रेय गुलाम मोहम्मद को है। इस नए वाद्य-यंत्र के आविष्कार का उद्देश्य बीन के पूर्ण आलापचारी को ऐसे वाद्य-यंत्र में सम्मिलित करना था जो बीन की तरह न तो पारम्परिक था, न पेचिदा। उस समय का प्रचलित सितार बहुत छोटा था और आशय के उपयुक्त नहीं था और इसी हेतु उस समय की मांग के अनुसार सुरबहार का आविष्कार हुआ था। इसके आविष्कारकर्ता उमराव ख़ां या गुलाम मोहम्मद थे, जानने योग्य विशेष बात यह है कि सुरबहार जैसे वाद्य का आविष्कार बीनकार घराने में हुआ था, जिससे आगे चलकर, आधुनिक सितार का विस्तार हुआ।

हाँलाकि शुरूआत में सुरबहार घराने के बाहर के विद्यार्थियों के लिए ही था किन्तु जैसे-जैसे इस यंत्र की ध्वनि गुणवत्ता तथा क्षमता बढ़ती गई और अपनी चरम सीमा पर पहुँच गई, सेनिया घराने के संगीतज्ञों ने भी इस वाद्य-यंत्र को बजाना शुरू कर दिया और इसमें महारत हासिल कर ली। किन्तु गुलाम मोहम्मद और उनके बेटे सज्जाद मोहम्मद ने जो महारत और प्रसिद्धि सुरबहार बजाने में प्राप्त की वो अद्वितीय थी।

डॉ. लालमणि मिश्र के अनुसार- ${ }^{1}$

सुरबहार का नामोल्लेख प्राचीन अथवा मध्यकालीन ग्रन्थों में उपलब्ध नहीं होता। वास्तव में सुरबहार की उत्पत्ति सितार के पश्चात् हुई है। सेनी घराने के वीणावादक कलाकार अपने घर के लड़कों के अलावा और किसी को अपने वाद्य की शिक्षा नहीं देना चाहते थे। कुछ ऐसे ही बाहरी शिष्यों के लिए उन्होंने लोक में प्रचलित यन्त्र (सितार) को अपनाया था किन्तु इस यन्त्र में वीणा के आलाप जोड़ का काम अच्छा नहीं होता था। अतएव कुछ रईस कला-प्रेमियों के द्वारा अभिव्यक्त उस इच्छा को, कि वे वीणा के आलाप का काम सीखना चाहते हैं, पूरी करने के लिए सेनी घराने के उस्तादों ने सितार को आकार में बड़ा कर उसमें मोटे तार लगाकर सुरबहार का नाम प्रदान किया। उक्त परिवर्तन से उसकी ध्वनि गम्भीर हो गयी तथा मींड का काम भी वीणा के समान होने लगा। सुरबहार की रचना यद्यपि सेनियों ने अपने खानदान से बाहर के लोगों की शिक्षा के लिए की थी किन्तु उसकी गम्भीर ध्वनि तथा वीणा की प्रत्येक विशेषता से युक्त होने के कारण इस वाद्य को खानदानी उस्तादों ने भी अपना लिया। फलतः सुरबहार के कई प्रसिद्ध वादक इस देश में हुए किन्तु गत दो-तीन दशकों में सितार ने ध्वनि की दृष्टि से ऐसी उन्नत अवस्था प्राप्त की जिससे सुरबहार की आवश्यकता ही लगभग समाप्त हो गयी। वर्तमान समय में सुरबहार वादन का प्रचार बहुत कम हो गया है।

डॉ. प्रकाश महाडिक के अनुसार- ${ }^{2}$

आवश्यकता ही आविष्कार की जननी है। सुरबहार का आविष्कार तत्कालीन आवश्यकता का ही परिणाम दिखायी देता है। श्री सुललित सिंह के अनुसार, "एक बार की बात है कि गुलाम मुहम्मद ने बड़े खेद के साथ कहा था कि उमराव ख़ां जैसे महान् बीनकार के शिष्य होते हुए भी उन्हें वीणा (बीन) की तालीम नहीं मिली.....12 वर्ष के लम्बे अरसे तक उन्हें केवल सितार गत आदि से ही संतुष्ट रहना पड़ा

1 डॉ. लालमणि मिश्र, भारतीय संगीत वाद्य, पृ. 250

2 डॉ. प्रकाश महाडिक, भारतीय संगीत के तंत्री वाद्य, पृ. 110

Swar Sindhu : A National Peer-Reviewed Journal of Music | 
जो उनके लिए दुर्भाग्य जनक विषय था उन्होंने (उमराव ख़ां) अपने शिष्य को यह कहकर आश्वासन दिया कि वे उन्हें (शिष्य को) बीन की तालीम अन्य किसी वाद्य यन्त्र में देंगे। इस घटना के पश्चात् उन्होंने लखनऊ के दक्ष कारीगरों को बुलाकर एक ऐसा वाद्य यन्त्र बनाने को कहा जिसका तुम्बा नीचे से चपटा तथा डन्डी चौड़ी हो। इसमें सात तारों का संयोग था क्योंकि डंडी चौड़ी होने से मीण्ड का कार्य बड़े सूक्ष्म रूप से प्रस्तुत करने में भी सुविधा मिली और लम्बी मीण्ड बजाने में भी सहायता मिलने लगी। उमराव ख़ां ने इस वाद्य यन्त्र का नाम सुरबहार रखा। ${ }^{2}$

श्रीपद् बन्द्योपाध्याय के अनुसार, "उमराव ख़ां जिन्हें ध्रुपद और वीणा का पूर्ण ज्ञान था। गुलाम मोहम्मद नामक एक शिष्य को जिसे वीणा सीखने की प्रबल इच्छा थी, उसे एक बहुत बड़ा सितार बनाकर दिया,जिसमें तरबें भी लगाई गई थी। उमराव ख़ां ने अपने शिष्य गुलाम मोहम्मद को इसी वाद्य पर वीणा के ढंग से आलाप, जोड़ और झाले का शिक्षण दिया। इसी यन्त्र को 'सुरबहार' कहते हैं। हमारे संगीत रत्न (हाथरस) में भी उमराव ख़ां को सुरबहार का निर्माता बताया गया है। ${ }^{4}$ श्री ग. ह. तारलेकर ने भी सुरबहार के आविष्कार का श्रेय उमराव ख़ां को ही दिया है। लेकिन डॉ एस परांजपे के मतानुसार, "गुलाम मोहम्मद को इसके आविष्कार का श्रेय दिया जाता है। एक अन्य मत के अनुसार प्रसिद्ध सितार वादक शहदाद ख़ां ने सितार में सुधार कर इस नए वाद्य सुरबहार का आविष्कार किया। ${ }^{6}$ श्री अरविन्द पारिख का भी यही मत है कि साहबदाद ख़ां (शहदाद खां) ने ही सुरबहार का आविष्कार किया था।

एस. कृष्णा स्वामी ${ }^{7}$ के अनुसार-

सुरबहार उत्तरी भारत का सबसे अधिक मनमोहक वाद्य यंत्र है। यह करीब 120 साल पहले बनाया गया था। सुरबहार असल में सितार का ही एक बड़ा रूप है। यह लकड़ी का बना हुआ होता है तथा इसका पिछला भाग चपटा होता है। इसके तार सितार के तार से मोटे होते हैं तथा इसी कारण इसे नीचे स्वर में मिलाया जाता है। इसे मिलाने तथा बजाने की तकनीक सितार की तरह होती है परन्तु इसकी ध्वनि बेहतर होती है। सुरबहार खासतौर से हिन्दुस्तानी शास्त्रीय संगीत में बजाने के लिए उपयुक्त है। सुरबहार पर आलाप, जोड़ तथा झाला ध्रुवपद अंग से बजाया जाता है। सुरबहार के आविष्कार का श्रेय प्रसिद्ध बीनकार उमराव ख़ां को है जिन्होंने अपने मुख्य शिष्य गुलाम मोहम्मद ख़ां को इसे बजाने की तकनीक सिखाई थी। गुलाम मोहम्मद ख़ां और उनके पुत्र सज्जाद मोहम्मद दोनों प्रसिद्ध सुरबहार वादक थे।

शर्मिष्ठा सेन के अनुसार ${ }^{8}-$

1 श्री सुललित सिंह, विश्व वीणा, भाग-1, पृ. 85

2 श्री सुललित सिंह, विश्व वीणा, भाग-1, पृ. 85

3 श्रीपद् बन्द्योपाध्याय, सितार मार्ग, तृतीय भाग, पृ. 102

4 लक्ष्मी नारायण, हमारे संगीत रत्न, पृ. 459

5 श्री ग. ह. तारलेकर, भारतीय वाद्यांचा इतिहास, पृ. 76

6 डॉ. ऍँ. परांजपे, संगीत वाद्य, पृ. 144-145

7 S. Krishnaswamy, Musical Instruments of India, Page-30

8 Sharmistha Sen, The String Instruments of North India, Page - 54, 56 
सुरबहार वाद्य बनावट में कच्छपी वीणा से थोड़ा अलग है। कच्छपी की तुलना में सुरबहार वाद्य की ध्वनि कोष आकार में बड़ा होता है। इसमें 7 खूंटियाँ होती हैं जिनमें सात धातु की तारें लगी होती हैं। इसमें तरब की तारें भी लगी होती हैं जिसकी खूंटियाँ थोड़ी छोटी होती हैं।

सुरबहार की पार्श्व तंत्रिकाएं या तरब की तारें तांबे की होनी चाहिए। इसमें दो तंत्र-शान (ठतपकहम) होते हैं जिसमें बड़ा तंत्र-शान मुख्य सात तारों को तथा दूसरा छोटा तंत्र-शान तरब की तारों को ग्रहण करता है। इस वाद्य को बजाने की तकनीक व इसकी बैठक कच्छपी जैसे ही होती है। सारिका विन्यास व पर्दों के साथ ही इसे मिलाने की विधि भी कच्छपी जैसी ही है। गम्भीर तथा मधुर ध्वनि उत्पादन के लिए वादक अपनी इच्छा अनुसार तरब की तारों को मिला सकते हैं। सुरबहार वाद्य में कच्छपी से अधिक समय तक आंस रहती है।

Allyn Miner के अनुसार ${ }^{1}-$

हिन्दुस्तान में 19 वीं शताब्दी के अंत में सुरबहार नामक एक प्रमुख वाद्य-यंत्र बनाया गया जो चपटा तुम्बा युक्त कच्छुआ सितार का ही वृह्द रूप था। इसकी गहरी और लम्बी ध्वनि और मीण्ड की क्षमता के कारण यह बीन की भाँति बजाया जाता है। सितार के इस वृह्द रूप का प्रारम्भिक उल्लेख कप्तान रॉबर्ट स्मिथ की डायरी में मिलता है। सन् 1828 और 1833 के मध्य स्मिथ ने कानपुर में एक संगीत सभा का चित्र बनाकर इसका वर्णन किया। स्मिथथ ने इसमें वादकों को ढोलक व इस वृह्द सितार का वादन करते हुए दर्शाया है। यह वृहद सितार प्रारम्भिक सुरबहार का ही एक स्वरूप हो सकता है। स्मिथ के इस चित्र में वादक के दाएं हाथ की स्थिति बहुत अजीब है जो भ्रामक लगती है।

सुरबहार का उद्गम, कानपुर के पास लखनऊ में सन् 1825 के लगभग हुआ। 'यन्त्र कोश' में एस. एम. टैगोर के अनुसार लखनऊ के गुलाम मोहम्मद ने लगभग 50 वर्ष पहले सुरबहार का प्रचार किया। ${ }^{2}$ सितार वादक गुलाम मोहम्मद, 19 वीं शताब्दी की शुरूआत में लखनऊ के जाने-माने बीन वादक उमराव ख़ां के शिष्य थे। करम इमाम ख़ां ने गुलाम मोहम्मद के बजाने की बहुत प्रशंसा की है किन्तु सुरबहार से उनके संबंध के बारे में कुछ नहीं कहा। उन्होंने गुलाम मोहम्मद के सितार पर बीन वादन की शैली (ठोंक) में निपुणता के बारे में बताया है।

गुलाम मोहम्मद ने सर्वप्रथम सितार में अद्भुत प्रभुत्व प्राप्त किया। उनके ठोंक में इतनी स्पष्टता ओर दक्षता थी जिसे मैंने उमराव ख़ां के अतिरिक्त किसी बीनकार या रबाबिए से नहीं सुना। उनका सितार किसी भी तरह बीन या रबाब से कम नहीं था। ${ }^{3}$

रहीम बेग ने अपनी पुस्तक 'नग़मा-ए-सितार' में सितार का चित्र पेश करते हुए लिखा था कि इसका आविष्कार गुलाम मोहम्मद ने किया था। इस वाद्य-यंत्र में पांच तार हैं, एक चौड़ी गर्दन, एक ऊपरी तुम्बा और दस तरब की तारें हैं किन्तु इसमें चिकारी नहीं है। एक अन्य चित्र में बताया गया है कि इस तरह के सितार गुलाम मोहम्मद की कृतियाँ हैं। एक पुरबिया और उस्ताद दोनों इसके वादन एवं निर्माण में निपुण थे। बनारस के एक शिल्पी हनुमान दयाल ने मेरी उपस्थिति में रीमा के राजा साहब के लिए

1 Allyn Miner, Sitar and Sarod in the 18th and 19th Centuries, Page - 55

2 Surinder Mohan TAgore, yantra Kosh, page - 34

3 मोहम्मद करम इमाम, मदन-उल-मौसिकी, पृ. 45 
इस प्रकार का सितार बनाया था। 19 वीं शताब्दी के उत्तरार्द्ध में, बंगाल, सुरबहार बजाने का मुख्य केन्द्र बन गया था जो सज्जाद हुसैन अथवा सज्जाद मोहम्मद के नाम से जुड़ा हुआ था। करम इमाम के अनुसार सज्जाद हुसैन, गुलाम मोहम्मद के बेटे थे। $2^{2}$ टैगोर के अनुसार सुरबहार, बड़ा कच्छुआ सितार (कच्छपी वीणा) से भिन्न नहीं है। सितार की भाँति इसमें सात तार हैं। इसकी तरबें (तरफ या पार्श्व तंत्रिका) अमूमन पीतल की बनी होती हैं। एक छोटा-सा ब्रिज (तंत्रासन) मुख्य ब्रिज के नीचे इन तारों को ग्रहण करता है। वादक की इच्छानुसार इन तारों को मिलाया जाता है। टैगौर के अनुसार सुरबहार को बिल्कुल, कच्छपी की भाँति पकड़ा व बजाया जाता है। इसकी ध्वनि गहरी, गम्भीर, मृदु और देर तक रहने वाली होती है। टैगोर का कहना है कि निपुण शिल्पी द्वारा निर्मित कच्छपी की ध्वनि सुरबहार से किसी भी प्रकार कम नहीं होगी। ${ }^{3}$ स्पष्टतः टैगौर इस नए वाद्य-यंत्र से ज्यादा प्रभावित नहीं थे।

विक्टर चार्ल्स महिलॉन की सन् 1880 के सूची-पत्र में इस यंत्र समूह का विवरण है, जो टैगोर ने ब्रूसलस के अजायबघर को दे दिया था। महिलॉन के विवरण में सुरबहार की लम्बाई 1.5 मीटर लिखी है। बंगाली किताबों में पाँच मुख्य तारों और दोनों चिकारी को मिलाने के दो ढंग इस प्रकार हैं -

म.(स्टील), स़(पीतल), प. (पीतल), प़(स्टील),स. (पीतल), स., प (स्टील की चिकारी)

म (स्टील), स़(पीतल), स़(पीतल), प(स्टील), स़. (पीतल), स, प (स्टील की चिकारी)

'नग़मा-ए-सितार' में गुलाम मोहम्मद के सितार में दस तरब की तारें वर्णित हैं इसकी संभावना है कि सितार में जो तरब के तार जोड़ें हैं वे गुलाम मोहम्मद तथा उनके नए सितार से प्रभावित हैं। 19वीं शताब्दी के अन्त में और 20 वीं शताब्दी की शुरुआत में, कलकत्ता के सितार निर्माता कन्हाई लाल ने जो सुरबहार बनाए वे बनावट में सितार से काफी भिन्न थे, जिसमें एक उभार रहित (समतल) पर्दा, चौड़ी गर्दन और एक सजावटी छिद्र में तरब के तार बन्धे हुए हैं, सम्मिलित हैं। सुरबहार में विशेष रूप से पतले व चपटे पर्दे लगाए गए हैं ताकि वह बीन के पर्दो की तरह लगें।

19 वीं सदी की किताबों में सुरबहार के बारे में कोई उल्लेख नहीं मिलता है। इस वाद्य का मुख्य उद्देश्य आलाप बजाना था, न कि राग (गत) जो बीन पर बजाते हैं। बीन का संगीत और आलाप की तकनीक पुस्तकों का विषय नहीं था क्योंकि ये व्यावसायिक संगीतकारों की जागीर मानी जाती थी, जिसे वे सीधे उस्तादों से सीखते थे। इस कारण सुरबहार अपेक्षाकृत विशेष वाद्य-यंत्र था और इसे अधिक प्रसिद्धि नहीं मिली परन्तु फिर भी इस वाद्य ने सितार वादकों और बीनकारों का ध्यान अपनी ओर खींचा।

संदर्भ ग्रंथ सूची

इमाम मोहम्मद करम (1856), मदल-उल-मौसिकी, हिन्दुस्तानी प्रेस, लखनऊ, प्रथम संस्करण, गर्ग लक्ष्मी नारायण (1978) हमारे संगीत रत्न, संगीत कार्यालय हाथरस, उत्तर प्रदेश, तृतीय संस्करण

Miner Allyn (1997), Sitar and Sarod in the 18th and 19th Centuries, Motilal Banarsidass Publishers Private Limited, Delhi First Indian Edition.

Tagore Surinder Mohan (1875), Yantra Kosh or A Treasury of the Musical Instruments of Ancient and of modern India and of various other countries, Reprint, New York: AMS Press, 1st Edition

\footnotetext{
1 रहीम बेग, नग़मा-ए-सितार, पृ. 53

2 मोहम्मद करम इमाम, मदन-उल-मौसिकी, पृ. 45

3 Surinder Mohan Tagore, yantra Kosh, page-35
} 